\title{
Análise das complicações clínico-obstétricas em gestantes adolescentes segundo a Classificação de Robson
}

\author{
Analyzing clinical obstetric complications in pregnant adolescents by the Robson Classification
}

Análisis de las complicaciones clínico-obstétricas en adolescentes embarazadas según la Clasificación de Robson

\author{
Mirelly Barbosa Cortez' ${ }^{\prime}$; Amuzza Aylla Pereira dos Santos ${ }^{\prime} \oplus$; Maria Elisângela Torres de Lima Sanches ${ }^{\prime} \mid \oplus$;

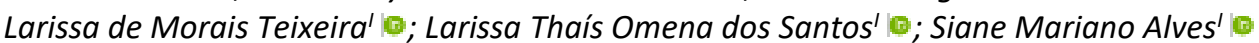

'Universidade Federal de Alagoas, Maceió, AL, Brasil

\begin{abstract}
RESUMO
Objetivo: analisar as complicações obstétricas de gestantes adolescentes por meio da Classificação de Robson. Método: tratase de uma pesquisa quantitativa documental. Foram pesquisados 150 prontuários de gestantes adolescentes de alto risco. 0 estudo foi de setembro a novembro/2019 e iniciou após a aprovação do Comitê de ética em Pesquisa da Universidade Federal de Alagoas. Resultados: as taxas de cesáreas do grupo 1 foram o dobro do recomendado (18,92\%), pela Classificação de Robson. No grupo 2, houve $100 \%$ de cesárea, enquanto recomendação é de 20 a 35\%. No grupo 4 observou-se $100 \%$ de parto vaginal, enquanto os grupos 5, 8 e 10 excederam o número de cesáreas em cerca de 15,40 a 20\%. Conclusão: evidenciou-se, por meio da Classificação de Robson, que o tipo de parto das gestantes adolescentes que foram afetadas por uma complicação clínicoobstétrica foi o parto cesáreo, com aumento nos grupos de gestante 1, 2, 5, 8 e 10.

Descritores: Enfermagem Obstétrica; Gravidez na Adolescência; Risco; Classificação.
\end{abstract}

\section{ABSTRACT}

Objective: to analyze obstetric complications in pregnant adolescents using the Robson Classification. Method: in this quantitative study, 150 medical records of high-risk adolescent pregnant women were searched between September and November 2019, after approval by the research ethics committee of Alagoas Federal University. Results: by the Robson Classification, cesarean section rates in group 1 were twice as high as recommended (18.92\%). Group 2 returned 100\% cesarean section, while the recommendation is 20 to $35 \%$; group 4 showed $100 \%$ vaginal deliveries; and, in groups 5,8 and 10 , these exceeded the number of cesarean sections by about 15.40 to $20 \%$. Conclusion: using the Robson Classification, it was shown that pregnant adolescents affected by a clinical obstetric complication were delivered by cesarean sections, which increased in groups 1, 2, 5, 8 and 10.

Descriptors: Obstetric Nursing; Pregnancy in Adolescence; Risk; Classification.

\section{RESUMEN}

Objetivo: analizar las complicaciones obstétricas en adolescentes embarazadas mediante la Clasificación de Robson. Método: se trata de una investigación cuantitativa documental. Se investigaron 150 historias clínicas de adolescentes embarazadas de alto riesgo. El estudio fue de septiembre a noviembre/2019 y se inició después de la aprobación del Comité de Ética en Investigación de la Universidad Federal de Alagoas. Resultados: Las tasas de cesáreas en el grupo 1 fueron el doble de lo recomendado (18,92\%) según la clasificación. En el grupo 2, hubo 100\% de cesáreas, mientras que la recomendación es del 20 al 35\%. En el grupo 4, el 100\% tuvo parto vaginal, mientras que los grupos 5,8 y 10 superaron el número de cesáreas en aproximadamente del 15,40 al 20\%. Conclusión: queda claro, a través de la Clasificación de Robson, que el tipo de parto de las adolescentes embarazadas que se vio afectado por una complicación clínico-obstétrica fue el cesáreo, con incremento en los grupos de embarazadas 1, 2, 5, 8 y 10.

Descriptores: Enfermería Obstétrica; Embarazo en Adolescencia; Riesgo; Classificación.

\section{INTRODUÇÃO}

Quando a gestação ocorre durante a adolescência, há uma série de implicações biológicas, psicológicas, sociais e econômicas, pois, mães adolescentes possuem maior risco de resultados sociais adversos e problemas de saúde de curto prazo ${ }^{1}$. Assim, o Ministério da Saúde, considera a idade materna como um dos determinantes de risco gestacional².

Gestantes adolescentes apresentam menor número de consultas de pré-natal e maiores índices de não comparecimento; maior frequência de recém-nascidos de baixo-peso e, além disso, a maior parte das gestações não são planejadas, o que pode acarretar maiores riscos de complicações e danos fetais, pela ocasional exposição materna a medicamentos, álcool e drogas ${ }^{2}$. Logo, as complicações que acometem essas mulheres implicam em resultados que poderão prejudicar a história obstétrica delas de forma permanente. Para exemplificar, no Brasil, em 2017, quase 40\% das adolescentes foram submetidas à cesárea ${ }^{3}$.

Autora correspondente: Amuzza Aylla Pereira dos Santos. E-mail: amuzza.santos@gmail.com Editora Científica: Cristiane Helena Gallasch 
Diante disso, é fundamental entender o comportamento dessas complicações obstétricas, e seus desfechos para as mães e seus filhos. Visto que, após uma cesárea prévia, os riscos são maiores para uma segunda cesárea ${ }^{4}$ e para hemorragia obstétrica, devido a elevação da probabilidade de ocorrência de deiscência e ruptura uterina ${ }^{5}$.

Desse modo, para compreender a relação entre as complicações de gestantes adolescentes e os desfechos obstétricos utilizou-se nesta pesquisa a Classificação dos Dez Grupos de Robson. Esse sistema, pela Organização Mundial da Saúde, deve ser usado em todo o mundo, como instrumento padrão com o objetivo de avaliar, monitorar e comparar as taxas de cesarianas ao longo do tempo e entre hospitais ${ }^{6}$.

Os grupos que compõem esse sistema de classificação baseiam-se em cinco parâmetros obstétricos: a história obstétrica, o estabelecimento do trabalho de parto, a posição fetal, o número de filhos e a idade gestacional, o que permite conhecer o perfil as gestantes mais submetidas ao parto cesárea7.

Nesse contexto, emergiu a seguinte questão norteadora: conforme a Classificação de Robson, como se dá o comportamento dos resultados obstétricos de gestantes adolescentes que foram acometidas por complicações? Dessa maneira, elencou-se como objetivo: analisar as complicações obstétricas de gestantes adolescentes, por meio da Classificação de Robson.

\section{REFERENCIAL TEÓRICO}

A gestação na adolescência e mortalidade materna estão associadas. No Brasil, em 2017, mais de $12 \%$ das mortes maternas foram de mulheres na faixa etária de 10 aos 19 anos $^{8}$, é necessário compreender, portanto, quais as causas e consequências que levam aos desfechos obstétricos desfavoráveis. Diante disso, torna-se fundamental conhecer os desfechos de parto dessas gestantes.

É válido ressaltar que o alto número de cesáreas em adolescentes é preocupante. O relatório "Nascer no Brasil", aponta que entre os anos 2007 e 2012, 42\% das adolescentes foram submetidas à cesariana ${ }^{9}$. Esse dado é alarmante, visto que, mulheres que iniciam a vida reprodutiva cedo, tendem a ter maior número de filhos e, logo, estarão expostas a mais riscos em gestações futuras ${ }^{10}$.

Esses dados demonstram que há um descompasso entre o cuidado prestado a parturientes adolescentes e o que recomenda o Ministério da saúde. Pois, deve-se garantir que a adolescente tenha acompanhante no pré-parto, parto e puerpério, visto que, a presença do acompanhante diminui a chance de ocorrer complicações. E, o estresse está relacionado ao prolongamento do trabalho de parto aumentando a chance de que a adolescente seja submetida a uma cesariana $^{10}$.

A cesárea é o ato cirúrgico que consiste em incisar a região abdominal e a parede do útero da gestante com o propósito de extrair o feto ali desenvolvido ${ }^{11}$. Essa intervenção cirúrgica é procedimento de eleição quando a vida da mãe e feto estão em risco. É fundamental monitorar as taxas de cesáreas em hospitais levando em consideração as características dessas mulheres durante a internação. A Classificação é simples, reproduzível, clinicamente relevante e, prospectiva. Assim, toda gestante internada pode ser classificada antes do parto em um dos 10 grupos $^{6}$.

A Classificação auxilia os hospitais a otimizar o uso de cesáreas ao identificar, analisar e focalizar intervenções em grupos específicos que sejam de relevância local e avaliar a eficácia de estratégias e intervenções criadas para otimizar o uso da cesariana ${ }^{5}$. Além disso, é imprescindível avaliar a qualidade da assistência, dos dados contidos, do cuidado clínico e os desfechos por grupo, bem como é fundamental que toda equipe multidisciplinar esteja atenta para importância desse registro ${ }^{6}$.

Percebe-se assim, a relevância em estudar quais grupos de mulheres têm parto cesáreo, especialmente quando se trata de adolescentes que apresentam a idade como fator de risco na gestação e, por isso, possuem mais chances de desenvolver uma complicação.

\section{MÉTOdo}

Trata-se de um estudo descritivo, retrospectivo e documental, de abordagem quantitativa. Realizado na maternidade Professor Mariano Teixeira do Hospital Universitário Professor Alberto Antunes no município de Maceió.

A pesquisa foi realizada com 150 prontuários. Os critérios de inclusão foram: prontuários localizados e identificados de gestantes adolescentes, dos 10 aos 19 anos, com complicações clínico-obstétricas, descobertas durante a gestação, nos anos de 2017 e 2018. Já, os critérios de exclusão: prontuários que faltavam informações relevantes à pesquisa, sendo considerados, assim, incompletos.

Para a coleta de dados foi construído um formulário estruturado com o objetivo de organizar os dados de identificação, a história obstétrica, as complicações e os desfechos de gestantes adolescentes. A partir de então, o 
projeto foi enviado a Comitê de Ética e Pesquisa da Universidade Federal de Alagoas, e após a aprovação, deu-se início à coleta dos dados.

A pesquisa foi desenvolvida sob as diretrizes da Resolução no 466 de 12 de dezembro de 2012, a qual aprova as normas para pesquisa envolvendo seres humanos, incorporando sob a ótica dos indivíduos e coletividades: referenciais da bioética, tais quais, a autonomia, não maleficência, beneficência, justiça e equidade, entre outros e visa assegurar os direitos e deveres que dizem respeito aos participantes da pesquisa, à comunidade científica e ao Estado.

O Comitê de Ética em Pesquisa aprovou em 10/09/2019 o protocolo no 3.606.169. E, assim, deu-se início à pesquisa dos dados. Sendo os riscos oferecidos mínimos, uma vez que, não houve contato com as participantes da pesquisa, assim como não Ihes trouxe benefícios.

Foi elaborado o Termo de Assentimento Livre e Esclarecido, e o Termo de Consentimento Livre e Esclarecido (TCLE) do responsável, por se tratar de pesquisa com menores de 18 anos, porém não foi necessário o uso durante a coleta de dados, pois, a pesquisa ficou restrita à análise documental e, assim, não houve contato com as participantes. Os dados foram tabulados e analisados no programa Microsoft Office Excel 2013, e depois foram criados gráficos, colunas e tabelas visando melhor visualização dos resultados.

\section{RESULTADOS E DISCUSSÃO}

\section{Perfil obstétrico das gestantes adolescentes}

Procurou-se entender o perfil das gestantes adolescentes acolhidas na maternidade de referência em alto-risco e que apresentaram alguma complicação obstétrica. Foi observado, que a maior parte das adolescentes se encontrava na primeira gestação, porém é importante destacar que uma pequena parcela já era secundigesta. Esses dados seguem a mesma linha de um estudo realizado no Estado de São Paulo, no qual, $48,3 \%$ estavam na primeira gestação e $25,6 \%$ apresentavam duas gestações ou mais. Destaca-se que o estudo supracitado classificou as gestantes secundigestas e tercigestas no mesmo grupo ${ }^{11,12}$.

Quanto à paridade, no presente estudo, a maioria das mulheres eram nulíparas e 13,33\% eram primíparas. Um perfil de paridade similar foi encontrado em um estudo feito na Nigéria, no qual, 94,12\% eram nulíparas e 5,33\% primíparas $^{13}$. No que concerne ao número de abortos dessas mulheres, o presente estudo apontou que uma parcela significativa já sofreu algum aborto, fato que merece atenção dos profissionais. Um estudo sobre abortamento na adolescência está associado ao desenvolvimento de distúrbios psiquiátricos após o evento. Isso demonstra que as adolescentes em situação de abortamento necessitam de um acolhimento de qualidade ${ }^{14}$.

\section{Complicações obstétricas diagnosticadas em gestantes adolescentes}

Com o objetivo de entender as complicações dessas gestantes e sua influência nos desfechos obstétricos, procurou-se conhecer as principais complicações que às acometem. Das complicações encontradas, três obtiveram destaque: as síndromes hipertensivas (17,33\%), seguidas do trabalho de parto prematuro (16,00\%) e a Doença Trofoblástica Gestacional (6,67\%).

Houve diferença do predomínio dessas complicações conforme a faixa etária. As adolescentes de 10 a 14 anos tiveram predomínio de trabalho de parto prematuro. As pertencentes à faixa etária de 15 a 19 anos, apresentaram predomínio das síndromes hipertensivas, conforme Figura 1.

As síndromes hipertensivas foram as complicações mais prevalentes, aspecto que confirma, essa patologia ser tida como uma causa importante de morbidade grave, incapacidade prolongada e morte de mães e bebês. Os distúrbios hipertensivos da gravidez são a primeira causa de morte materna em todo o mundo ${ }^{15}$. Desse modo, torna-se claro, que é um problema que deve ser investigado em gestantes adolescentes.

A outra complicação em destaque, neste estudo, foi o trabalho de parto prematuro. A literatura é quase unânime em afirmar que a associação da gestação na adolescência e o aspecto socioeconômico e cultural que ela está inserida são fatores de risco determinantes para que a prematuridade e o baixo peso ocorram ${ }^{16}$. Uma análise efetuada no Estado do Acre apresentou que ocorreram 626 partos prematuros na cidade de Rio Branco, destes $25 \%$ eram de adolescentes entre 10 e 19 anos. Isso expressa que as adolescentes representam parcela significativa desses casos ${ }^{17}$.

Seguida dessas complicações, a Doença Trofoblástica Gestacional também apareceu em número significativo. Porém, ressalta-se que no Estado de Alagoas, há um único Hospital de referência para a ocorrência de doença trofoblástica gestacional, que foi o local desta pesquisa. Em recente trabalho que compara centros de referência sulamericanos e norte-americanos, a porcentagem de gestação molar foi mais de 30\% em adolescentes na América Latina, e na América do Norte foi de $13 \%{ }^{18}$. Dos 2190 casos atendidos em 32 anos no Centro de Doença Trofoblástica Gestacional da Irmandade Santa Casa de Misericórdia de Porto Alegre, 25\% foi de adolescentes ${ }^{19}$. 


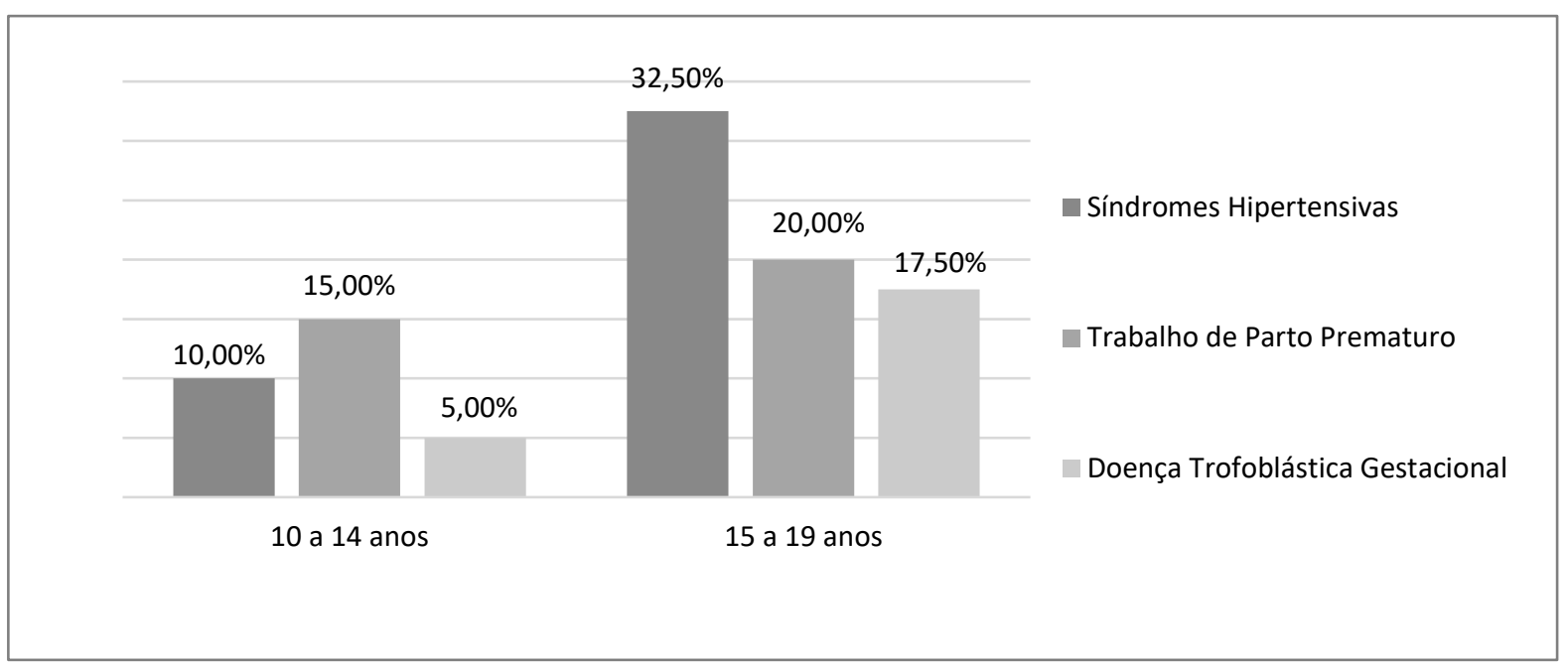

FIGURA 1: Principais complicações de gestantes adolescentes atendidas em maternidade de alto risco por faixa etária de 10 a 14 anos e 15 a 19 anos, no período de 2017 a 2018. Maceió, AL, Brasil, 2019.

As outras complicações clínico-obstétricas encontradas apresentaram valores abaixo de $6 \%$ do total das gestantes participantes desta pesquisa. Essas complicações foram: vulvovaginites, infecção do trato urinário, ruptura prematura de membranas ovulares, distúrbio psiquiátrico, descolamento prematuro de placenta, malformação fetal. Além dessas, a aloimunização rh, cardiopatia materna, pielonefrite, distúrbios hematológicos, diabetes mellitus, oligoidrâmnio, restrição de crescimento intrauterino, epilepsia, anemia falciforme, cistite, corioamnionite, crise convulsiva, distócia de parto, diabetes mellitus gestacional, aborto, hepatite viral C, hematoma em vulva, hidronefrose, vírus da imunodeficiência humana, neoplasia intraepitelial cervical, nódulo miomatoso e óbito fetal, também apresentaram baixo percentil neste estudo ${ }^{18,19}$.

\section{Análise dos desfechos obstétricos, segundo a Classificação de Robson, de gestantes adolescentes que apresentaram complicações obstétricas}

Diante das complicações apresentadas, foram investigados, neste estudo, os desfechos maternos e fetais dessas adolescentes acometidas por problemas na gestação. Essa análise, faz-se de grande importância, visto que, a cesariana está associada a maior chance de morte materna e infecção pós-parto ${ }^{20}$. Quanto ao tipo de parto, metade teve sua resolução por meio de parto vaginal e metade por cesárea.

No que se refere aos fatores associados à cesariana entre primíparas adolescentes, aquelas com nível de escolaridade adequado para a idade, as na adolescência mais tardia e classe econômica mais elevada têm maior proporção de parto cesáreo. Já aquelas da classe C, D e E têm número de cesáreas reduzido ${ }^{19}$.

O tipo de parto no presente estudo concorda com a afirmação, uma vez que as mulheres de faixa etária mais tardia (15 aos 19 anos) foram mais submetidas à cesárea, enquanto que as da faixa etária mais jovem (10 aos 14 anos) tiveram o parto vaginal, em sua maior parte. Das gestantes estudadas, a maior parte foi classificada nos grupos 1 e 10 da Classificação de Robson. Não houve gestantes que se enquadrassem nos grupos 3, 7 e 9 de Robson. As do grupo 1 são aquelas nulíparas, com feto único, cefálico, maior ou igual a 37 semanas, em trabalho de parto espontâneo ${ }^{5}$. Essas tiveram maior número de partos vaginais (81,08\%). Resultado que concorda com as taxas nacionais brasileiras, uma vez que, no ano de 2017, as gestantes na faixa dos 10 aos 19 anos, e classificadas no grupo 1, tiveram 68\% de partos vaginais em todo o Brasil ${ }^{21}$.

A Organização Mundial da Saúde organizou as interpretações de Robson para cada taxa de cesáreas, nessa sistematização há recomendações sobre qual deve ser a taxa de cesáreas para cada grupo. Para o grupo 1, é destacado que taxas menores que $10 \%$ são possíveis. Porém, nas gestantes deste estudo, o número de cesáreas foi quase o dobro $(18,92 \%)$ que o esperado pelas recomendações de Robson. O outro grupo encontrado foi o grupo 2 , nulíparas com feto único, cefálico, maior ou igual a 37 semanas, cujo parto é induzido ou que são submetidas à cesárea antes do início do trabalho de parto ${ }^{6}$, dessas todas foram submetidas à cesárea. As taxas brasileiras, das gestantes adolescentes classificadas no grupo 2 , apontam que $50 \%$ foi submetida ao parto cesáreo ${ }^{21}$. 
Com isso, pode-se perceber que a complicação obstétrica levou a todas as gestantes adolescentes do grupo 2 serem submetidas à cesárea. De acordo com as recomendações de interpretação de Robson, o grupo 2 apresenta taxa de cesárea consistentemente de 20 a $35 \%^{22}$. Na presente pesquisa, percebe-se que todas as gestantes foram submetidas à cesárea, o que demonstra uma alta taxa, para aquela que é esperada.

As do grupo 4, são as multíparas sem cesárea anterior, com feto único cefálico maior ou igual a 37 semanas em trabalho de parto espontâneo ${ }^{6}$, todas tiveram parto vaginal. Os dados brasileiros são parecidos com os encontrados nessa pesquisa, pois, no ano de 2017, 67,26\% das gestantes adolescentes do grupo 4 tiveram parto vaginal ${ }^{22}$. Demonstrando que, apesar de terem tido complicações, as adolescentes participantes dessa pesquisa, do grupo 4 , apresentaram mais partos vaginais.

Conforme a Classificação de Robson, as gestantes do grupo 4 que passam por cesariana, raramente chegam aos $15 \%{ }^{21}$. Neste estudo, todas apresentaram parto vaginal, percebe-se, assim, que nesse grupo, as taxas foram aquelas esperadas. $\mathrm{O}$ grupo 5 são todas as multíparas com pelo menos uma cesárea anterior, com feto único cefálico, maior ou igual a 37 semanas $^{6}$, todas desse grupo foram submetidas à cesárea. No Brasil, as gestantes adolescentes desse grupo, em sua maioria, também foram submetidas à cesariana (75,60\%). Faz-se necessário, ressaltar que 23,41\%, do grupo 5, em todo o Brasil, apesar de apresentarem uma cesárea anterior, tiveram parto vaginal ${ }^{19}$.

A recomendação para o grupo 5 , é de 50 a $60 \%$ de cesáreas ${ }^{22}$. No presente estudo, todas tiveram sua resolução de parto por meio de cesarianas, estando, desse modo, aquém do esperado. As gestantes classificadas no grupo 6, são todas as nulíparas, com feto único em apresentação pélvica ${ }^{6}$. No presente estudo, todas tiveram parto cesáreo. Enquanto que, no Brasil, 83,29\% tiveram parto cesáreo, porém, mais de 15\% apresentou parto vaginal, apesar de seus fetos estarem em apresentação pélvica ${ }^{21}$. O Manual de recomendação não aponta critérios de interpretação dos dados de cesárea para o grupo $6^{22}$.

Houve, também, gestantes classificadas no grupo 8, todas as mulheres com gestação múltipla incluindo aquelas com cesáreas anteriores, e todas foram submetidas à cesárea ${ }^{6}$. Esse achado é semelhante aos dados brasileiros, que apresentaram mais de $70 \%$ das gestantes adolescentes no grupo 8 submetidas à cesárea. Porém, é importante ressaltar que $26,22 \%$ teve parto vaginal ${ }^{21}$. Para o grupo 8 , a recomendação, usualmente é de $60 \%$ de cesáreas ${ }^{19}$. Na presente pesquisa, todas foram submetidas à cesárea, demonstrando, novamente, taxa mais alta que a esperada.

Já as do grupo 10, são todas as gestantes com feto único cefálico < 37 semanas, incluindo aquelas com cesáreas anteriores $^{6}$. Essas tiveram maior número de cesarianas $(81,08 \%)$. Esse dado demonstrou que as gestantes classificadas no grupo 10, que apresentaram alguma complicação e foram atendidas em maternidade de alto risco foram mais submetidas à cesárea.

No Brasil, no ano de 2017, as gestantes adolescentes, do grupo 10, tiveram mais partos vaginais (68,29\%) do que partos cesáreos 0 grupo 10 apresenta, normalmente, taxas de $60 \%$ de cesáreas ${ }^{20}$. Nesta pesquisa, a porcentagem de cesáreas desse grupo foi de $20 \%$ mais alta que o recomendado pela Classificação, isso aponta que as complicações clínico-obstétricas que acometem as gestantes adolescentes desse grupo, levaram-nas a ter mais partos cesáreos, o que pode trazer ainda mais complicações em gestações futuras. Por conseguinte, fica evidente a importância de entender os Grupos de Robson e suas maiores chances de parto cesáreo, entre as adolescentes. Pois, as complicações que elas apresentam aumentam o risco de cesariana na maior parte dos grupos desta classificação ${ }^{21}$.

Assim, a compreensão, da importância da atenção por parte dos profissionais aos grupos de Robson é fundamental. Uma vez que, conhecendo a classificação do grupo de Robson que aquela gestante ou parturiente faz parte, será possível atuar de forma preventiva com vistas a diminuir a ocorrência de uma cesárea desnecessária. Pois, os dados apresentados expressam que, as adolescentes pesquisadas apresentaram mais partos cesáreos do que os números de todo o Brasil e do que os índices indicados ideais pela Classificação de Robson. Desse modo, para a elevação da qualidade da atenção obstétrica, é imprescindível, a utilização dessa Classificação. Esse sistema oportuniza um conhecimento melhor da história obstétrica da adolescente, e com isso uma contribuição à qualidade de assistência prestada ${ }^{21,22}$.

\section{CONCLUSÃO}

Ao analisar os resultados, observa-se que as complicações obstétricas em gestantes adolescentes por meio da Classificação de Robson mais recorrente foram as do grupo das Síndromes Hipertensivas, seguida de trabalho de parto prematuro e Doença Trofoblástica Gestacional, e com relação ao desfecho, percebe-se que os grupos 1,2,5,8 e 10 por terem sofrido uma complicação clínico-obstétrica, aumentou o número de partos cesáreos. Isso demostra que a atenção à saúde das adolescentes deve ser intensificada, uma vez que, as complicações podem aumentar o percentual do número de cesárea recomendada pela classificação, levando em risco o futuro obstétrico dessas mulheres, bem como as taxas de morbimortalidade, proporcionando desfechos desfavoráveis para as adolescentes gestantes. 


\section{REFERÊNCIAS}

1. Xavier C, Benoit A, Brown HK. Teenage pregnancy and mental health beyond the postpartum period: a systematic review. J Epidemiol Community Health [Internet], 2018 [cited 2020 Mar 07]; 72(6):451-457. DOI: https://doi.org/10.1136/jech-2017209923.

2. Ministério da Saúde (Br). Instituto Sírio-Libanês de Ensino e Pesquisa. Protocolos de Atenção Básica - Saúde das mulheres. Brasília (DF): Ministério da Saúde; 2016. [cited 2020 Mar 07]; Available from: http://bvsms.saude.gov.br/bvs/publicacoes/protocolos_atencao_basica_saude_mulheres.pdf.

3. Ministério da Saúde (Br). Nascimentos por residência da mãe por tipo de parto segundo região. Brasília (DF): Ministério da Saúde; 2017. [cited 2020 Mar 07]; Available from: http://tabnet.datasus.gov.br/cgi/tabcgi.exe?sinasc/cnv/nv.uf.def.

4. Fundação Oswaldo Cruz. Principais Questões sobre Parto Vaginal Após Cesariana (PVAC - VBAC). Rio de Janeiro; 2019. Available from: https://portaldeboaspraticas.iff.fiocruz.br/atencao-mulher/principais-questoes-sobre-parto-vaginal-apos-cesariana-pvacvbac/\#: :text=Depois\%20de\%20uma\%20ces\%C3\%A1rea\%2C\%20os,nas\%20chances\%20de\%20parto\%20vaginal.

5. Vääräsmäki M, Raudaskoski T. Pregnancy and delivery after a cesarean section. Duodecim [Internet], 2017 [cited 2020 Mar 07]; 133(4):345-52. Available from: https://pubmed.ncbi.nlm.nih.gov/29205981/.

6. Organização Mundial da Saúde (OMS). Departamento de Saúde Reprodutiva e Pesquisa. Declaração da OMS sobre Taxas de Cesáreas. Geneva (SWZ); 2015.

7. Kindra, T. Análise das indicações de cesáreas com base na classificação de dez grupos de Robson em uma maternidade pública de risco habitual [Doctoral dissertation]. Curitiba: Universidade Federal do Paraná; 2017.

8. Ministério da Saúde (Br). Departamento de Informática do Sistema Único de Saúde. Óbitos maternos por Faixa Etária det segundo Região. Brasília (DF): Ministério da Saúde; 2017. [cited 2020 Mar 07]. Available from: http://tabnet.datasus.gov.br/cgi/tabcgi.exe?sim/cnv/mat10uf.def.

9. Fundação Oswaldo Cruz. Escola Nacional de Saúde Pública. Relatório: “Nascer no Brasil”. Brasília (DF); 2012.

10. Instituto da Infância (IFAN). Rede Nacional da Primeira Infância. Primeira Infância e Gravidez na adolescência. Secretaria Executiva (IFAN) Biênio (2013/2014). Fortaleza (CE); 2014

11. Montenegro CAB, Rezende FJ. Obstetrícia fundamental. Rio de Janeiro: Editora: Guanabara Koogan; 2018.

12. Okuda GT, Cavalhieri FB, Pereira ACS, Danno CH, Takeda E, Stasi GGD. Social and obstetric profile of pregnant adolescent women. Ciênc. cuid. Saúde [Internet], 2017 [cited 2020 Mar 07]; 16(2). DOI: https://doi.org/10.4025/cienccuidsaude.v16i2.28455.

13. Ijarotimi OA, Biobaku OR, Loto OM, Orji EO. Obstetric outcome of teenage pregnancy and labour in Obafemi Awolowo University Teaching Hospitals complex, Ile-Ife: A ten-year review. Tropical J Obstet Gynaecol [Internet]. 2019 [cited 2020 Mar 07]; 9(36):10511. DOI: https://doi.org/10.4103/TJOG.TJOG_13_19.

14. Lainscek FGT, Carlotto SDS, Carvalho RA, Chiacchio FBB, Amaral LROG. Adolescente: aspectos emocionais frente ao aborto. Revista CEREUS [Internet]. 2019 [cited 2020 mar 07]; 11(4):72-83. DOI: https://doi.org/10.18605/2175-7275/cereus.v11n4p72-83.

15. Farias RV, Soares CFS, Araújo RS, Almeida RSA, Leitão DS, Santos JS et al. Gravidez na adolescência e prematuridade: uma revisão integrativa de literatura. REAS [Internet]. 2020 [cited 2020 Mar 07]; 56:1-10. Available from: https://acervomais.com.br/index.php/saude/article/view/3977.

16. Organização Mundial da Saúde. Organização Panamericana de Saúde. Folha Informativa - Mortalidade Materna. Brasília (DF); 2018.

17. Hydall ARS, Duarte RN, COSTA, RSL. Partos prematuros em adolescentes em Rio Branco -Acre no ano de 2015. Dêciência em Foco - Uninorte [Internet]. 2018 [cited 2020 Mar 07]; 1(2):34-44. Available from: http://revistas.uninorteac.com.br/index.php/DeCienciaemFoco0/article/view/144\#: :text=Resultados\%3A\%200s\%20resultado s\%20apontam\%20que,entre\%2020\%20e\%2049\%20anos.

18. Soares RR, Maetsál, Colón J, Braga A, Salazar A, Charry RC, Sun, SY, Goldstein DP, Bergowizt RS. Complete molar pregnancy in adolescents from North and South America: Clinical presentation and risk of gestational trophoblastic neoplasia. J Gynecol Oncol. [Internet]. 2016 [cited 2020 Mar 07]; 142(3):496-500. DOI: https://doi.org/10.1016/j.ygyno.2016.07.002.

19. Gama SGN, Viella EF, Schilitz AOC, Filha MMT, Carvalho ML, Gomes KRO, Costa MCO, Leal MC. Fatores associados à cesariana entre primíparas adolescentes no Brasil, 2011-2012. Cadernos de Saúde Pública [Internet]. 2014 [cited 2020 Mar 07]; 30[1]:11727. Available from: https://www.scielo.br/scielo.php?script=sci_arttext\&pid=S0102-311X2014001300018.

20. Mascarello KC, Horta BL, Silveira MF. Complicações materna e cesáreas sem indicação: revisão sistemática e meta-análise. Rev Saude Publica [Internet]. 2017 [cited 2020 mar 07]; 51:105. Available from: https://www.scielo.br/pdf/rsp/v51/pt_0034-8910rsp-S1518-87872017051000389.pdf.

21. Ministério da Saúde (Br). Departamento de Informática do Sistema Único de Saúde. Nascim p/resid. mãe por Grupos de Robson segundo Tipo de parto. Brasília (DF); 2017.

22. Organização Mundial da Saúde. Robson Classification: Implementation Manual. Geneva (SWZ); 2017. 